\title{
Activation Problem
}

National Cancer Institute

\section{Source}

National Cancer Institute. Activation Problem. NCI Thesaurus. Code C133631.

Problem associated with the activation of a device. 